\title{
Influence of bottom boundary condition on heat distribution within oxidizing heaps
}

\author{
J. Crawford* Y. A. Stepanyants*
}

(Received 26 October 2004, revised 6 October 2005)

\begin{abstract}
Different types of boundary conditions for temperature (Dirichlet, Neumann, mixed and some others) at the base of a heap comprising oxidizable material are numerically examined for their influence on temperature distribution and overall heat content within the heap. We demonstrate that some global heap characteristics (for example, the total heat content) essentially depend on boundary conditions and corresponding temperature distributions within the heap, whereas other global characteristics (for example, the overall oxidation rate) are not affected significantly. Practical recommendations on selection of boundary conditions are presented.
\end{abstract}

*Australian Nuclear Science and Technology Organisation, Menai (Sydney), Lucas heights, NSW 2234, Australia. mailto:Yury.Stepanyants@ansto.gov.au

See http://anziamj.austms.org.au/V46/CTAC2004/Craw for this article, (C) Austral. Mathematical Soc. 2005. Published October 20, 2005. ISSN 1446-8735 


\section{Contents}

1 Introduction

C1105

2 Traditional models of boundary conditions

C1108

2.1 Dirichlet boundary condition . . . . . . . . . . . C1108

2.2 Neumann boundary condition . . . . . . . . . . . . . . C1111

2.3 Mixed boundary condition . . . . . . . . . . . . C1111

3 Artificial boundary condition

C1114

4 Extended 2D model

C1116

5 Discussion

C1117

6 Conclusions and recommendations

C1122

References

C1123

\section{Introduction}

Mathematical models of processes occurring in piles of oxidizing sulfidic materials usually comprise a combination of physical equations based on fundamental principles and empirical relationships between physical variables $[6$, e.g.]. These relationships contain many fitting parameters which depend on the particular type of porous material.

Additional model uncertainty is introduced by inaccurate formulation of boundary conditions (BCs), particularly the formulation of the BC for temperature at the base of the heap. Measurements of temperature in the aforementioned piles show that due to heat release in the oxidizing material, the temperature within piles varies in space and time. A characteristic time scale for temperature to reach a quasi-equilibrium state for a dump of 15 to $20 \mathrm{~m}$ 
high comprising typical dump material is about 1.5 to 2.5 years [8]. In the majority of cases, the temperature at the base of the heap differs from that at the surface. The temperature at the surface is regulated by atmospheric conditions and is usually approximated either by yearly mean value or by monthly averaged values. The temperature of the ground immediately underlying the heap is expected to be equal to the temperature at the base of the heap.

In modelling the temperature distribution in a heap, it seems natural to take into account temperature continuity at the base of the heap. However, this requires either knowledge of the base temperature and heat flux at the interface between the heap and ground, or taking into consideration some part of a ground slab beneath the dump. The former case is usually unachievable due to lack of information about the required parameters. The latter case brings additional inconvenience due to the necessity to complicate the geometry of the considered problem and increase the computational domain which takes additional computer resources and is time costly. Therefore when modelling, either a condition on temperature or heat flux across the bottom boundary is applied for the sake of simplicity.

The most frequently used $\mathrm{BC}$ at the base of the heap is a condition of constant temperature equal to the ambient temperature in the atmosphere (Dirichlet BC). This usually leads to an underestimation of total heat content in the heap (see Section 5). In addition, calculations show that the temperature abruptly decreases near the heap base and approaches the ambient atmospheric value. Such a temperature distribution does not correspond to reality. The thickness of this transient layer where the temperature abruptly decreases from the maximum to ambient value varies from tens of centimeters to several meters depending on intrinsic heap parameters.

Numerical simulations conducted with another type of BC, when the temperature gradient is zero at the bottom (Neumann BC) [4, e.g.] also result in unrealistic temperature distribution within the heap with the temperature maximum usually occurring at the heap base. As shown below, this 
leads to the significant overestimation of the total heat content within the oxidizing heap.

To reach a better agreement between numerical results and observations, a mixed $\mathrm{BC}$ could also be used. As reported in many textbooks [2, e.g.], such a BC usually gives good results. But the drawback of this case is that the coefficient of proportionality between the heat flux and temperature is a priori unknown and must be chosen empirically. Implementation of such a BC has not yet been considered in the context of oxidizing heap modelling, and the appropriate coefficient of proportionality has never been estimated.

In order to have some understanding on the influence on model predictions resulting from inappropriately formulating the bottom BC for temperature, there is a necessity to investigate the effect of different types of BCs on temperature distribution within oxidizing heap, total heat content within the heap, and some other global heap characteristics, for example, global oxidation rate (GOR) of waste-rock dump [6].

In this article the problem described is studied systematically using a mathematical model of an oxidizing heap [6]. The finite-difference code SULFIDOX [1] was used for the numerical simulations of a $2 \mathrm{D}$ model of a heap of a trapezoidal cross-section. The modelled heap was $15 \mathrm{~m}$ high and $200 \mathrm{~m}$ wide at the base with the angle $45^{\circ}$ between the base and side. Numerical mesh dimensions were $31 \times 401$ nodes resulting in a spatial step $h=0.5 \mathrm{~m}$ in both the vertical and horizontal directions. Transient simulation was carried out with the time step $\Delta t=0.000005$ year $\approx 2.6$ min. It was assumed that the heap is comprised of uniform sulfidic material with the gas permeability of $K=10^{-9} \mathrm{~m}^{2}$ and the intrinsic oxidation rate (IOR) of $S=5.72 \cdot 10^{-8} \mathrm{~kg}(\mathrm{~S}) \mathrm{m}^{-3} \mathrm{~s}^{-1}$. The BCs for all variables are described in $[1,6]$, here we focus only on the $\mathrm{BC}$ for temperature at the base of the heap.

Dirichlet, Neumann and mixed BCs were examined in detail as well as a new artificial condition accounting for continuity of non-zero heat flux from the heap into the underlying ground. Results obtained for all these BCs 
are compared with the results of a numerical simulation of the extended 2D model of the heap overlying a ground slab. The last case is considered here as the most advanced and reliable model which includes both the heap and some portion of ground with the natural conditions of temperature and heat flux continuity at the heap-ground interface.

\section{$2 \quad$ Traditional models of boundary conditions}

Traditionally used BCs for temperature in the numerous university textbooks are particular cases of the mixed type $\mathrm{BC}$

$$
a \partial_{z} T-b\left(T-T_{\mathrm{amb}}\right)=0,
$$

where $a$ and $b$ are some fitting coefficients. This equation reduces in two extreme cases either to the Dirichlet (when $a=0$ ) or to the Neumann (when $b=0)$ BC.

\subsection{Dirichlet boundary condition}

Let us consider first the simplest case when the temperature at the base, $z=0$, is equal to a constant value, $T_{\mathrm{amb}}=15^{\circ} \mathrm{C}$, which is the ambient temperature for both the atmosphere and the ground. It is assumed that initially the temperature distribution is uniform across the heap and is equal to ambient temperature, that is, $T(0, \mathbf{r})=T_{\mathrm{amb}}$, where $\mathbf{r}$ is the radius-vector in the plane $(x, z)$. Heat is released during oxidation of the heap material, and temperature redistributes in accordance with the heat equation containing source and sink terms.

The dominant temperature transport mechanisms in a heap are affected by heap geometry and material intrinsic parameters. Usually diffusive transport occurs within heaps when the temperature slowly diffuses from the 
heated areas towards the external heap boundaries. This was the case of our study. The oxidation process was not stationary in general although in the middle part of the heap quasi-stationary conditions occurred for a relatively large time interval.

Figure 1-I shows temperature isotherms at three instants of time: (due to symmetry about the center only the left half of the heap cross-section is presented). In this figure, as well as in the subsequent figures, the difference between the temperature in the heap and ambient temperature, $\Delta T=T-$ $T_{\text {amb }}$ is presented. The temperature reaches its maximum, $\approx 33^{\circ} \mathrm{C}$, at about 2.5 years in "hot spots" positioned at lateral distances of about $20 \mathrm{~m}$ from the lower left and right edges of the heap and a height of $5 \mathrm{~m}$ from the bottom. Apparently, due to abundant oxygen supply both from the heap batters and top, the most intensive chemical reaction of oxidation occurs near the heap corners. This results in relatively fast temperature increase at these locations. Then, the "hot spots" move progressively towards the center of the heap as the oxidizable material is consumed at the edges. The zone of maximum temperature follows the region with highest oxidation rate. At about 4.2 years the maximum temperature, located at about $25 \mathrm{~m}$ (laterally) into the heap, begins to decrease. In the meantime, at the center of the heap, quasi-stationary temperature conditions set in for a relatively long time.

Figure 1-II shows the temperature profiles at different instants of time in the central vertical cross-section of the heap (solid curves) and the vertical cross-section at a distance of $x=25 \mathrm{~m}$ from the lower edges of the heap (dashed lines). The temperature reaches its maximum in the bulk of the heap approximately near the half depth for the chosen set of parameters and then, decreases to the ambient value at the bottom. The thickness of the bottom layer, where the temperature decreases, is approximately $5 \mathrm{~m}$. 

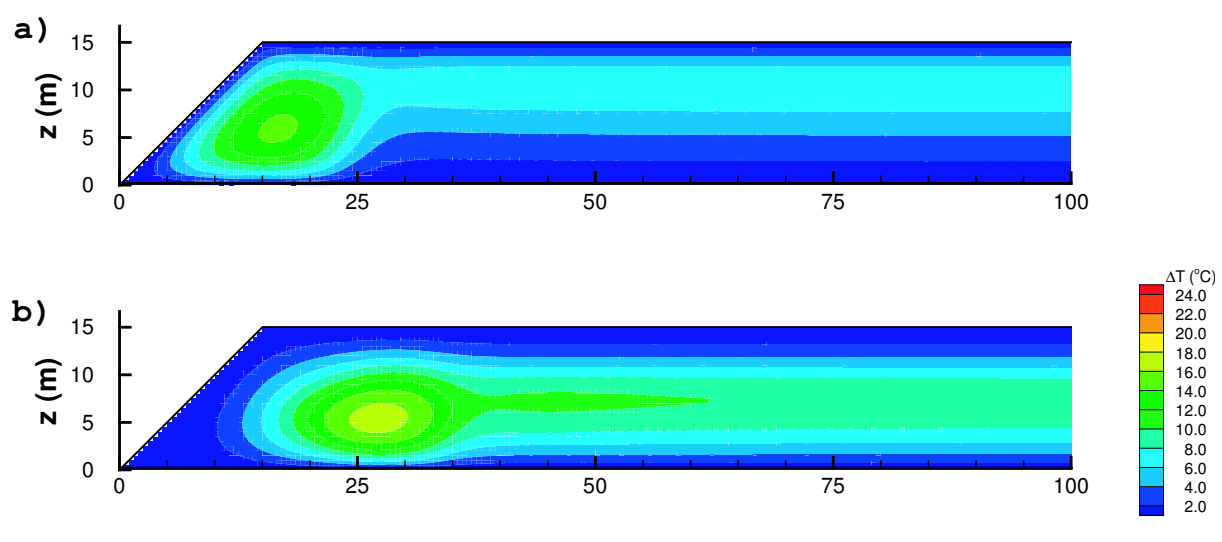

I
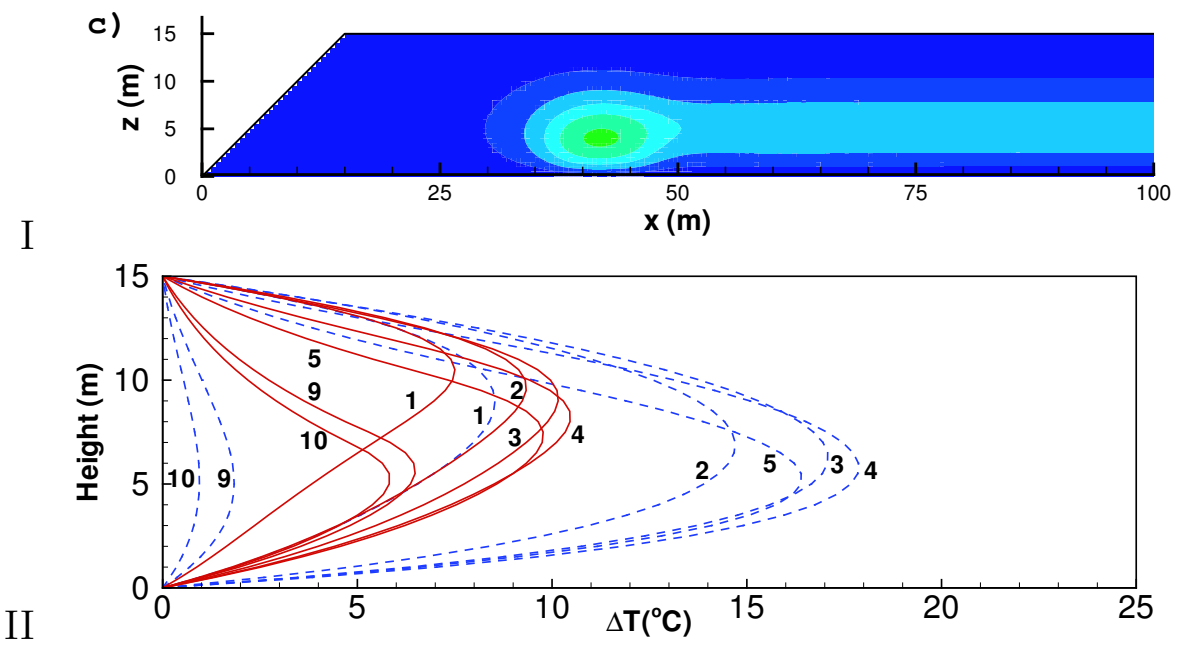

Figure 1: I. Temperature distribution in a heap with the Dirichlet BC at the base: a) $T=1 \mathrm{yr}$; b) $T=5 \mathrm{yr}$; c) $T=10 \mathrm{yr}$. II. Temperature profiles within the heap (labels of curves designate time in years). 


\subsection{Neumann boundary condition}

For the next studied case the temperature gradient at the heap base was zero $(b=0$ in Equation (1)). The calculated temperature distributions at the same three instants of time as before are shown in Figure 2-I.

The temperature reaches its maximum, $\approx 39^{\circ} \mathrm{C}$, in about three years, at $20 \mathrm{~m}$ (laterally) from the edges of the heap just at the interface with the ground. Then, this maximum slowly drifts to the center along the interface and begins to decrease after 5.6 years. At the center of the heap a quasistationary temperature distribution, when the amount of the released heat in a certain domain is equal to that diffusing from the domain, sets in again for a relatively long time. Figure 2-II shows the temperature profiles at different instants of time in the same vertical cross-sections of the heap as in Figure 1II. See in this figure that the temperature reaches its maximum in the bulk of the heap first and, then, the maximum gradually drifts to the heap base.

Such temperature behavior does not seem realistic because there are no physical reasons to consider the ground underneath the heap as an ideal insulator. Physical properties of the ground material (density, porosity, heat conductivity, specific heat capacity, etc) do not differ considerably from the properties of the heap material. Therefore, the heat flux at the bottom of the heap is expected to be finite rather then zero and directed to the ground (the heat is produced in the bulk of the heap and diffuses outside).

\subsection{Mixed boundary condition}

When the mixed BC, Equation (1), is used, the problem of adequate relationship between the coefficients $a$ and $b$ arises. Generally, there is no prior information to assist with selection of these coefficients. One can either rely on intuition or select values by fitting to experimental data. We examined different coefficient ratios $a / b$ and found that the case $a=10 b$ provides fairly 

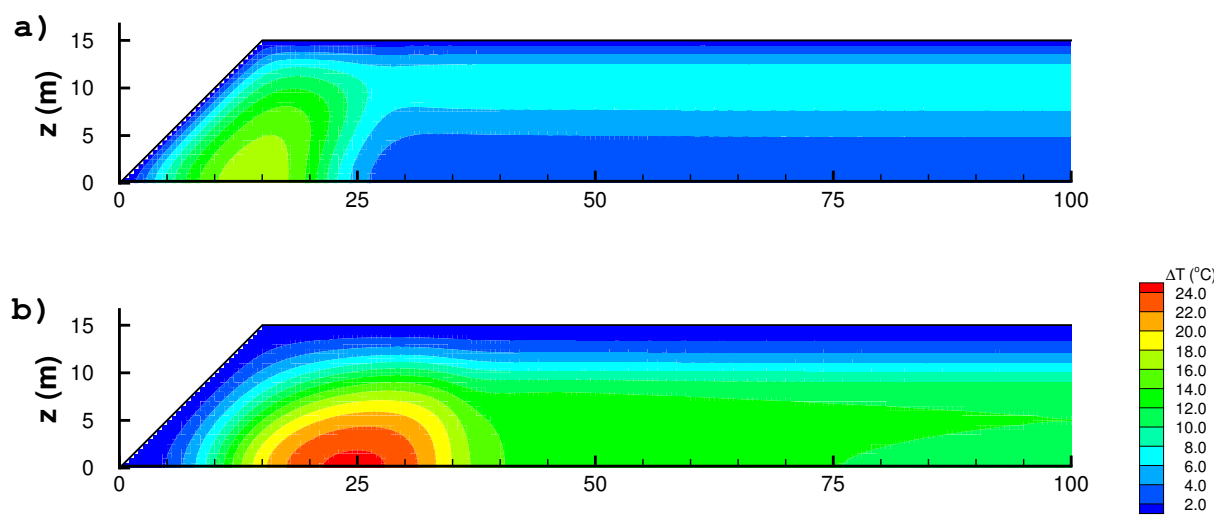

I
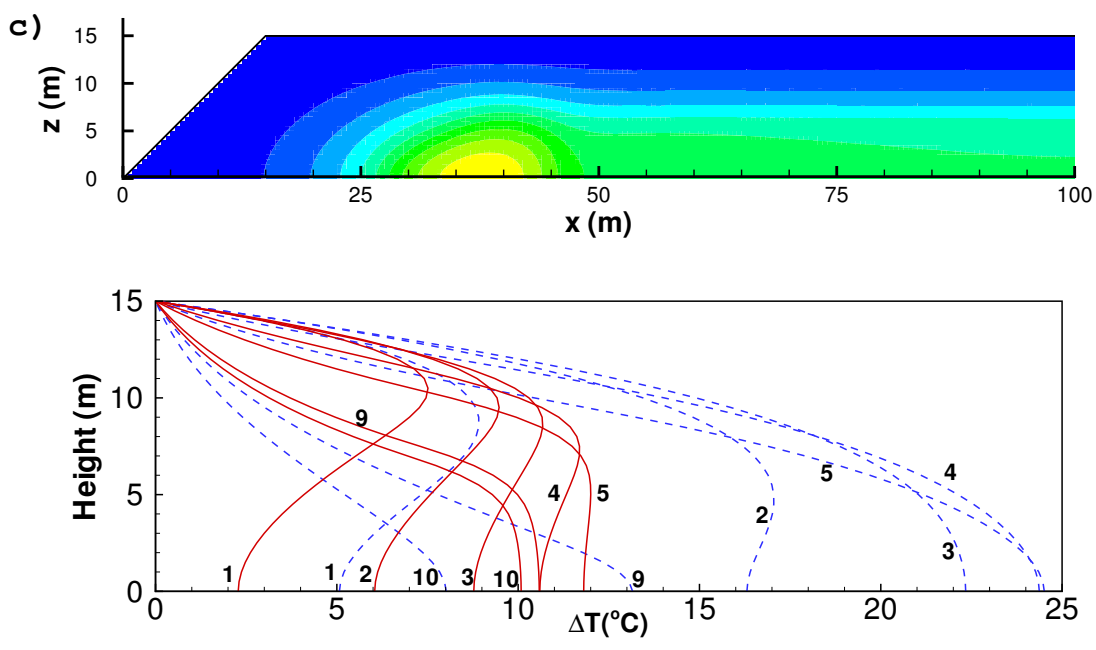

Figure 2: The same as in Figure 1 but for the Neumann BC at the heap base. 

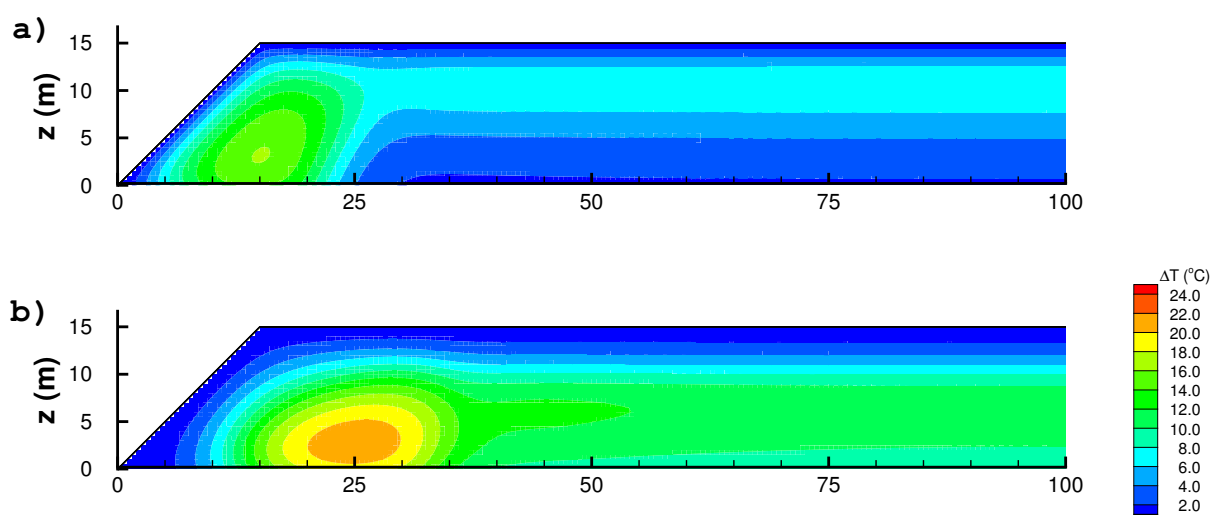

I
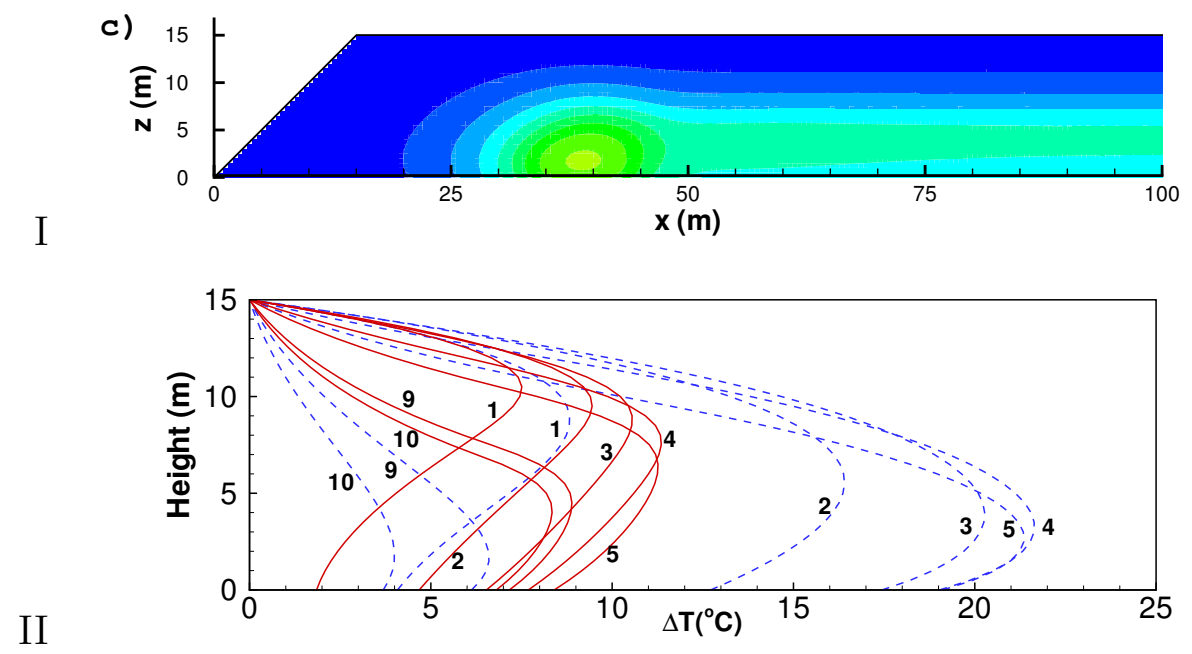

FiguRE 3: The same as in Figures 1 and 2 but for mixed BC with $a=10 b$. 
good agreement with the more complex representation described in Sections 3 and 4. Results obtained for this case are shown in Figure 3. In general, a mixed $\mathrm{BC}$ leads to some intermediate temperature distribution within the heap compared with the two extreme cases presented above for Dirichlet and Neumann BCs.

\section{$3 \quad$ Artificial boundary condition}

An alternative approach to set up a $\mathrm{BC}$ for temperature distribution in the restricted domain conjugated with another infinite region of different thermal properties was suggested by Vinsome and Westerveld [11] for the onedimensional case. The temperature distribution in the ground beneath the heap is presumed known and described by a simple trial function:

$$
T(t, x, z)=\left[\theta(t, x)+p(t, x) z+q(t, x) z^{2}\right] e^{z / d(t)}+T_{\infty},
$$

where $\theta(t, x)=T(t, x, 0)-T_{\infty}$ and $T_{\infty}$ is the temperature at "infinity" beneath the heap. Other parameters of this function, $p(t, x), q(t, x)$ and $d(t) \sim \sqrt{t}$, are determined by means of BC at the interface between the heap and ground, viz., from the condition of continuity of temperature and heat flux [11]. It is assumed also that the physical properties of the ground, that is, density, specific heat capacity, thermal conductivity, etc, are known. The temperature at the interface was also assumed to be a known function of time but not dependent on spatial coordinates.

For application to our problem, we extended this approach by modifying the BC. We assumed that the temperature at the base of the heap is not uniform but varies in time and space in accordance with heat transport processes occurring within the heap. Thus, the temperature at each point along the base of the heap must be matched with its own $z$-dependent trial function. Such a model can be called quasi-two-dimensional. Numerical computations 

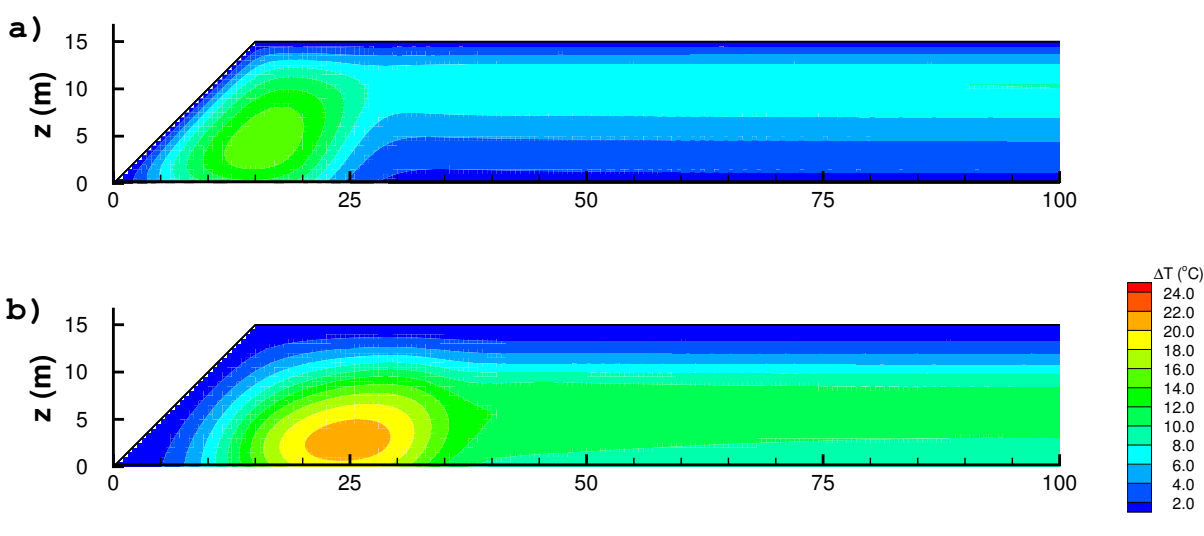

I
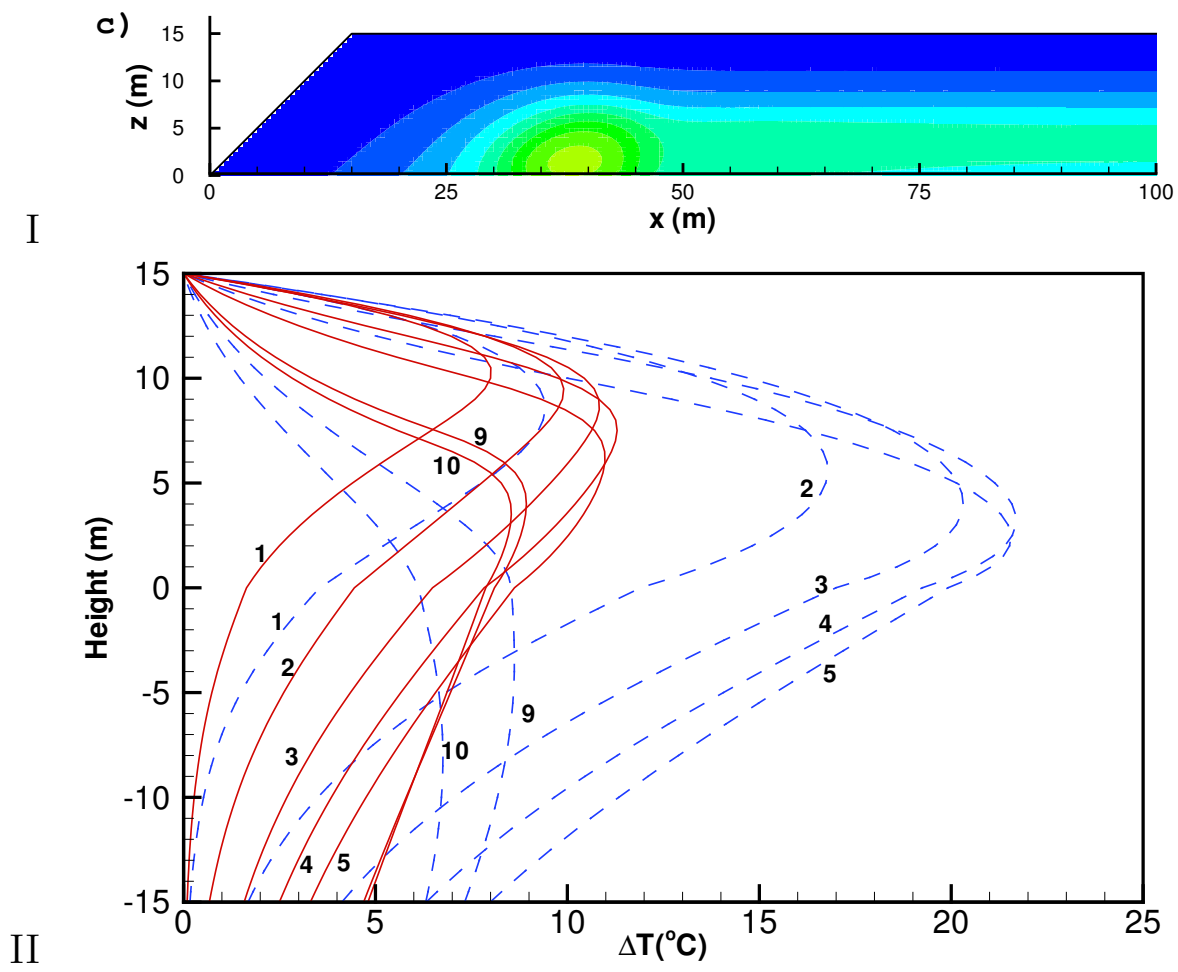

Figure 4: The same as in Figures 1-3 but for artificial BC. Temperature profiles in the frame II are shown both within the heap $(z>0)$ and ground $(z<0)$. 
were carried out on the basis of this model, the temperature pattern shown in Figure 4-I was obtained.

The temperature reaches its maximum, $\approx 37^{\circ} \mathrm{C}$, in about 2.8 years within two hot spots located at $19.5 \mathrm{~m}$ from the lower edges of the heap at $z=3.5 \mathrm{~m}$. These spots slowly drift to the near bottom region and move towards the heap center along the heap-ground interface. After 5.6 years the temperature within the hot spots is beginning to decrease. At the center of the heap a quasi-stationary temperature distribution sets in, as usual for a relatively long time. Temperature profiles are shown in Figure 4-II for different times. The virtual temperature distribution in the ground layer beneath the heap is also presented in that figure.

At each cross-section of the heap the temperature reaches a local maximum and then smoothly decays towards the bottom of the heap, through the heap/ground interface and then down to greater depths in the ground below the heap.

\section{Extended 2D model}

To test the validity of results obtained for the different cases studied, numerical simulations were undertaken including a more complex representation of the heap and the underlying ground slab. The modelled slab extended past the heap base to overcome the influence of slab BC. Except for the heap-slab interface, the temperature at all other outer slab boundaries is assumed constant and equal to $T_{\mathrm{amb}}$. Within the slab only the heat equation is used with matching conditions for temperature and heat flux at the interface between the heap and slab. This model is also approximate because only a finite rectangular portion from the semi-infinite ground was taken into consideration. However, by adjusting the free geometrical parameters, thickness and width of the slab, one can study the influence of constant temperature at the slab boundaries on the temperature behavior within the heap. Given that 
temperature decays with distance from the heated region, one might expect that the influence of fixed temperature at the outer slab boundaries becomes insignificant as the slab thickness and width increase.

Two simulations with different values of slab thickness $D$ and slab extension over the heap base size $l$ were conducted. For the first run $D=H$ and $l=0$, while for the second run $D=2 H$ and $l=15 \mathrm{~m}$ were used. The results are shown only for the second run (see Figure 5) which is treated as a reference case because the biggest portion of the ground is taken into consideration and, hence, we consider this model as closest to reality.

In both cases the evolution of temperature maxima and heat distribution within the heap are very similar. The temperature reaches its maximum, $\approx 36^{\circ} \mathrm{C}$, in about 3.5 years within two hot spots located at $21 \mathrm{~m}$ from the lower edges of the heap at $z=4 \mathrm{~m}$. The spot's evolution is qualitatively the same as was described before.

It was found that the influence of slab thickness on temperature distribution within the heap is small, especially in the early stages of heap oxidation. As expected, a greater difference arises in the corners of the heap, and slowly diffuses then into the bulk of the heap, reaching a maximum of $\Delta T=0.8^{\circ} \mathrm{C}$. After that, the temperature difference eventually decreased as the oxidized material is exhausted. The relative temperature difference did not exceed $0.25 \%$ and is mainly manifested in a thin bottom layer of the heap.

\section{Discussion}

The numerical code SULFIDOX has been modified to include the modelling of a portion of the underlying ground into the computational domain. The heat equation is considered in the rectangular ground slab with fixed temperature at the bottom and side boundaries, while at the interface between the heap and slab, conditions of temperature and heat flux continuity are applied. 
a)

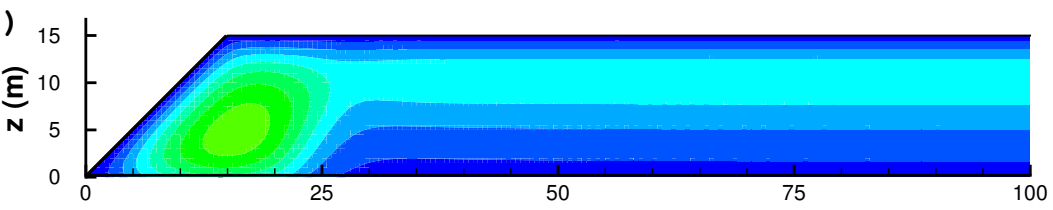

b)

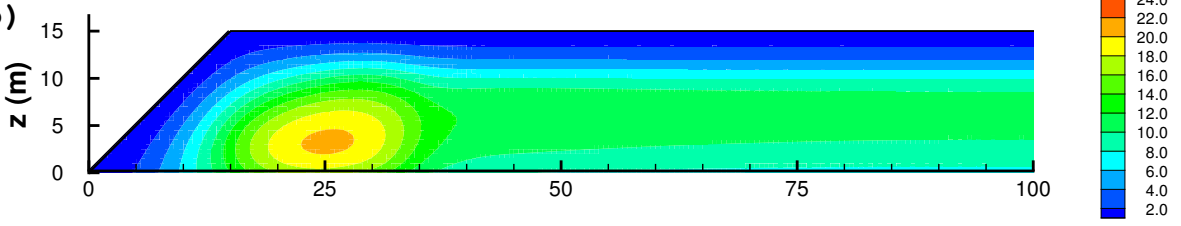

I
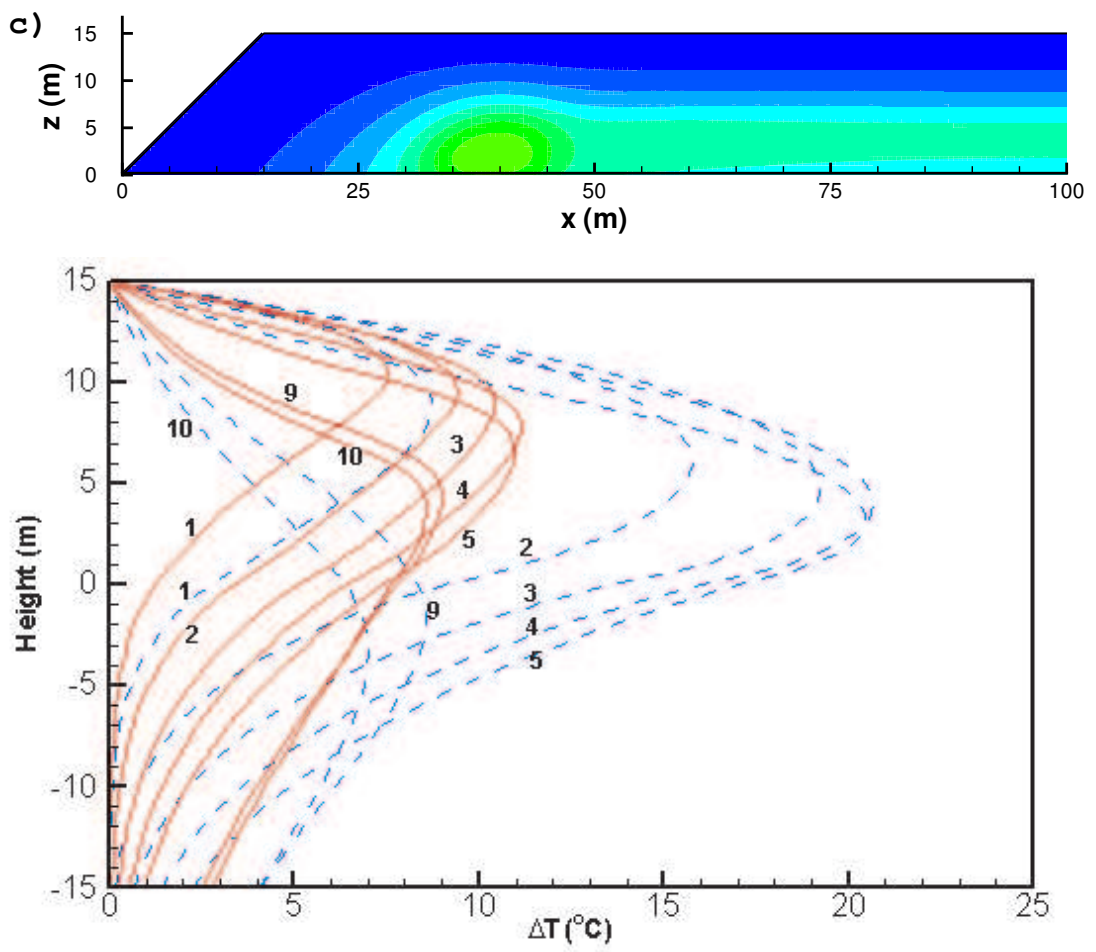

II

Figure 5: The same as in Figs. 4 but for extended 2D model with $D=2 H$ and $l=15 \mathrm{~m}$. 
Inclusion of the slab into the computations, while making the problem more complex and increasing computational time (only by 10\% approximately), can provide more reliable results and good agreement with the observed temperature distribution within heaps [3, 7, e.g.]. A detailed comparison of observed and calculated temperature distributions for particular waste-rock dumps could be a challenge for the future studies.

Numerical calculations demonstrated that the temperature distribution and heat regime within heaps strongly depend on the bottom BC. One of the global characteristics of an oxidizing heap is the total heat content $Q$. It is determined for a vertical heap slice of a unit thickness as

$$
Q(t)=\int_{A}\left(c_{g} \varepsilon_{g} \rho^{g}+c_{w} \varepsilon_{w} \rho^{w}+c_{s} \varepsilon_{s} \rho^{s}\right)\left[T(t, \mathbf{r})-T_{\mathrm{amb}}\right] d \mathbf{r},
$$

where $A$ is the cross-sectional area of the heap, $c_{\alpha}$ is the specific heat capacity of the $\alpha$-phase ( $\alpha=g, w, s$ for gas, water and solid phase, respectively), $\rho^{\alpha}$ is the intrinsic density of $\alpha$-phase, and $\varepsilon_{\alpha}$ is the volumetric content of $\alpha$-phase. The values of $Q(t)$ were calculated for all cases considered and plotted in Figure 6.

The total heat content is a time dependent characteristics of a heap. It increases due to heat release by chemical reactions at the beginning of heap oxidation when the oxidizing material is fresh. Then, it reaches a maximum (in approximately four years for the parameters chosen) and then, gradually decreases when the heap material is exhausted.

As expected, Dirichlet and Neumann BCs represent two extreme cases: the total heat content is least for the Dirichlet $\mathrm{BC}$, whereas it is greatest for the Neumann BC. All other curves for heat content are located between these two extreme curves. For the most sophisticated and realistic 2D case when the ground slab of thickness $D=2 H$ was taken into account, the heat content curve is considered as the reference case (Curve 7). Curve 6, calculated for the similar 2D case with a smaller thickness $D=H$ is indistinguishable from Curve 7. Curve 5, obtained for the artificial $\mathrm{BC}$ within the framework of 


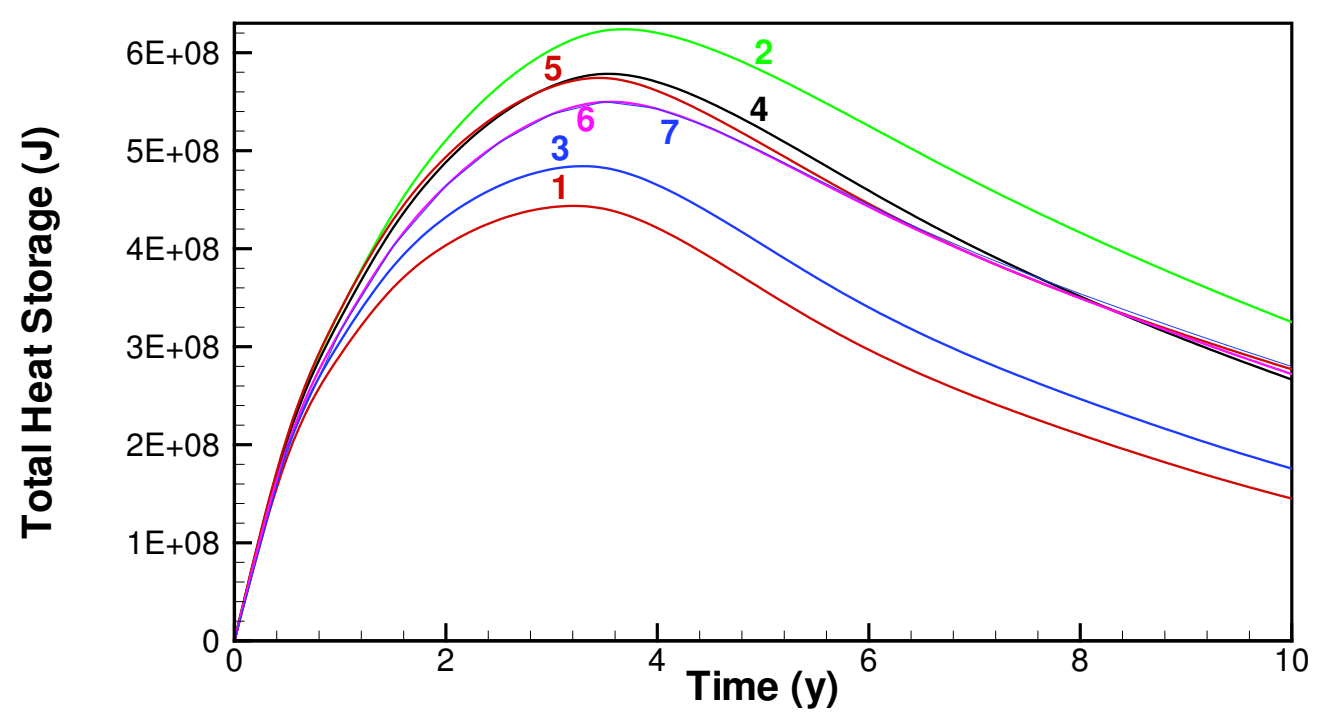

Figure 6: Total heat content in the heap versus time for different BCs: 1, Dirichlet BC; 2, Neumann BC; 3, mixed BC $(a=b) ; 4$, mixed BC $(a=10 b)$; 5 , artificial BC (pseudo-2D model); 6 , extended 2D model with the slab of thickness $D=H$ and zero extension; 7, extended 2D model with the slab of thickness $D=2 H$ and extension $l=15 \mathrm{~m}$ (the reference case). 
artificial pseudo-2D model, is also not far from the reference Curve 7 . The same can be said about Curve 4 for mixed BC with $a=10 b$. For a more appropriate relationship between coefficients $a$ and $b$, it might have been possible to obtain a curve closer to the reference Curve 7 . Using results obtained for the reference case, we tried to solve an inverse problem, that is to determine the appropriate value of the coefficient ratio $a / b$. Unfortunately, our simulation showed that the relationship between the coefficients depends both on time and position along the heap bottom in general.

Another important global characteristics of a heap is the overall (or global) oxidation rate of sulfur (GOR) which represents the rate of oxidation of reactive sulfur in a vertical column of unit lateral area per second [6]. The GOR is directly related to the primary pollutant generation rate $[3,10$, e.g.]. In our study the GOR was calculated as a function of time for each type of bottom BCs. It was discovered that there is a very little difference in the GOR values for different BCs, at least for the indicated set of parameters. This means that the GOR is not sensitive to details of temperature distribution within the heap while the temperature does not exceed some threshold value $\left(T_{\text {sick }} \approx 40^{\circ} \mathrm{C}\right)$ when the oxidation rate begins gradually decreasing and completely ceases at $T_{\text {kill }} \approx 60^{\circ} \mathrm{C}$ [6]. In our calculations with the chosen values of gas permeability and IOR, the hottest domains within the heap had a temperature of about $55^{\circ} \mathrm{C}$, and the domain sizes were relatively small (see Figures 1-4). Hence, only in those two small hot domains do temperature effects inhibit the oxidation rate. Within the rest of the heap the oxidation rate is independent of temperature. As the contribution of the small hot domains to GOR is minimal, the different BCs do not affect the outcome. This supports earlier results obtained by many authors to numerical calculations of GOR using simple BCs (Dirichlet or Neumann) at the base of the heap $[4,5,10$, e.g. $]$.

The effect of the BC on GOR has also been studied with higher values of IOR, in particular, for $S=8.81 \cdot 10^{-6} \mathrm{~kg}(\mathrm{~S}) \mathrm{m}^{-3} \mathrm{~s}^{-1}$ and other parameters remained unaltered. The highest temperature, $\approx 73^{\circ} \mathrm{C}$, was obtained for the 
Neumann BC, but again only within two restricted domains. The contribution of these domains to the GOR was not significant. Comparison of GOR dependency on time for the three different cases: Dirichlet and Neumann BCs as well as the extended 2D model shows that for all these cases the GOR is the same.

\section{Conclusions and recommendations}

Thus, the Dirichlet and Neumann BCs for temperature at the bottom of the heap are, apparently, the simplest both from theoretical and computational point of view. However, they represent extreme cases in terms of temperature distributions, that is, the total heat content within the heap is least for the Dirichlet and greatest for the Neumann BCs. Meanwhile, there is no noticeable difference in the GOR when these BCs (as well as others) are used.

The use of the mixed BC, Equation (1), does not significantly increase the complexity of the code or the computational cost. If the relationship between the coefficients $a$ and $b$ is chosen carefully, this BC can provide very good results for temperature distribution within the heap and for time dependent global characteristics of the heap (total heat content, GOR, etc).

The artificial quasi-2D model with a virtual temperature distribution in the ground beneath the heap can also provide good results. However, the computation cost is increased. The numerical code required significant modification to include the preliminary theoretical development of the algorithm. In addition, we found that implementation of the algorithm almost doubled the computational time. So, we conclude that this kind of $\mathrm{BC}$ has no advantages compared to the mixed $\mathrm{BC}$ with appropriate values for the coefficients $a$ and $b$.

The most accurate results were obtained using the advanced 2D model, when the ground slab beneath the heap was taken into account with given 
temperature at its boundaries. This model required modification of the algorithm to include a solution of the heat equation for the slab. However, implementation of the modified code was straightforward, and increased the computational time by only about $10 \%$ in comparison to the case of Dirichlet or Neumann BC. Moreover, the results obtained were very similar for models with zero extension and with finite $(15 \mathrm{~m})$ extension. The thickness of the slab is also unimportant if it is equal to or greater than the heap height.

To conclude : in spite of a good correlation between results obtained for different models, a detailed comparison of theoretical/numerical results on temperature distributions within particular waste-rock dump with experimental data is highly desirable. This will provide some validation of the models and, in addition, this could contribute to a more accurate solution to an important inverse problem of heat source distribution on the basis of data on temperature measurements [3].

One more issue needs future resolution: the dependency of IOR on the activation energy [9, 6, e.g.] was ignored in this study. However, in some cases the Arrhenius factor which accounts for intensification of chemical reactions with temperature at nonzero activation energy should be taken into account. The effect of activation energy on the temperature dependency of GOR might be crucial, and thus the GOR might depend on bottom BC in the heap.

\section{References}

[1] P. L. Brown et al. SULFIDOX: Version 1.1. A tool for modelling the temporal and spatial behaviour of heaps containing sulfidic minerals. ANSTO Report, ANSTO/ED/TN01-03, 2001. C1107

[2] W. B. Hall. Reactor Heat Transfer, Temple Press, London, 1958. C1107 
[3] J. R. Harries and A. I. M. Ritchie. The use of temperature profiles to estimate the pyritic oxidation rate in a waste rock dump from an opencut mine. Water, Air, and Soil Pollution, 15:405-423, 1981. C1119, C1121, C1123

[4] G. Pantelis and A. I. M. Ritchie. Macroscopic transport mechanisms as a rate-limiting factor in dump leaching of pyritic ores. Appl. Math. Model., 15:136-143, 1991. C1106, C1121

[5] G. Pantelis and A. I. M. Ritchie. Rate-limiting factors in dump leaching of pyritic ores. Appl. Math. Model., 16:553-560, 1992. C1121

[6] G. Pantelis, A. I. M. Ritchie, and Y. A. Stepanyants. A conceptual model for the description of oxidation and transport processes in sulfidic waste rock heaps. Appl. Math. Model., 26:751-770, 2002. http://dx.doi.org/10.1016/S0307-904X(01)00085-3 C1105, C1107, C1121, C1123

[7] S. M. Pidsley (Ed.). Rum Jungle Rehabilitation Project. Monitoring Report 1993-1998, Department of Infrastructure, Planning and Environment, Technical Report No. 01/2002, 2002. URL C1119

[8] A. I. M. Ritchie. The waste-rock environment. In: Short Course Handbook on Environmental Geochemistry of Sulfide Mine-Wastes, eds J. L. Jambor and D. W. Blowes, Mineralogical Association of Canada, Nepean, Canada, 22:133-161, 1994. C1106

[9] A. I. M. Ritchie. Optimization of biooxidation heaps. In: Biomining: Theory, Microbes and Industrial Processes, ed. by D. E. Rawlings, Springer, 201-226, 1997. C1123

[10] A. I. M. Ritchie and P. Miskelly. Geometric and physico-chemical properties determining sulfide oxidation rates in waste rock dumps. In: Proc. Fifth Int. Conf. Acid Rock Drainage, ICARD-2000, Denver, Colorado, 277-287, 2000. 
http://technology. infomine.com/hydromine/papers/icard4.pdf C1121

[11] P. K. W. Vinsome and J. Westerveld. A simple method for predicting cap and base rock heat losses in thermal reservoir simulators. The J. Canad. Petrol. Tech., July-September, 87-90, 1980. C1114 